\title{
A three-dimensional, two-way, parabolic equation model for acoustic backscattering in a cylindrical coordinate system
}

\author{
Zhu, Dong; Jensen, Leif Bjørnø
}

Published in:

Acoustical Society of America. Journal

Link to article, DOI:

$10.1121 / 1.1286074$

Publication date:

2000

Document Version

Publisher's PDF, also known as Version of record

Link back to DTU Orbit

Citation (APA):

Zhu, D., \& Jensen, L. B. (2000). A three-dimensional, two-way, parabolic equation model for acoustic backscattering in a cylindrical coordinate system. Acoustical Society of America. Journal, 108(3), 889-898. https://doi.org/10.1121/1.1286074

\section{General rights}

Copyright and moral rights for the publications made accessible in the public portal are retained by the authors and/or other copyright owners and it is a condition of accessing publications that users recognise and abide by the legal requirements associated with these rights.

- Users may download and print one copy of any publication from the public portal for the purpose of private study or research.

- You may not further distribute the material or use it for any profit-making activity or commercial gain

- You may freely distribute the URL identifying the publication in the public portal 


\title{
A three-dimensional, two-way, parabolic equation model for acoustic backscattering in a cylindrical coordinate system
}

\author{
Dong Zhu ${ }^{\mathrm{a})}$ and Leif Bjørnø \\ Department of Industrial Acoustics, Technical University of Denmark, Building 425, DK-2800 Lyngby, \\ Denmark
}

(Received 5 January 1999; accepted for publication 20 March 2000)

\begin{abstract}
A new PE model for solving three-dimensional, forward and backward sound propagation in a cylindrical coordinate system is presented. The model marches a wave field in the radial direction including the azimuthal diffraction effects, and solves for a backscattered field based on a three-dimensional, single scattering approach. A periodic sidewall boundary condition is applied for computations in a 360-degree sector, while an approximate sidewall boundary condition is used for calculation in a sector less than 360 degrees. These two sidewall boundary conditions are verified by the numerical results. The major drawback of using the cylindrical coordinate system, when the backscattering solution is valid within a limited area, is analyzed using a geometrical-optical interpretation. The model may be useful for studying three-dimensional backscattering phenomena comprising azimuthal diffraction effects. (c) 2000 Acoustical Society of America.
\end{abstract}

[S0001-4966(00)00607-X]

PACS numbers: 43.20.Bi, 43.30.Bp [DLB]

\section{INTRODUCTION}

Acoustic backscattering is an important issue in underwater acoustics, particularly in active sonar applications. However, it is normally difficult to model the backscattering in the ocean accurately, because fully three-dimensional effects and two-way propagation are involved. In recent years, the parabolic equation (PE) method ${ }^{1}$ has been widely used to solve various sound propagation problems including acoustic backscattering. Backscattering from scatterers of arbitrary shape in three dimensions in a Cartesian coordinate system has been recently solved using the PE technique combining a direct computation of the scattered field based on nonhomogeneous boundary conditions on the scattering objects. ${ }^{2}$ In this method the size of objects can range from a few wavelengths to hundreds of wavelengths. Another earlier method for solving three-dimensional backscattering in a Cartesian coordinate system was developed on the basis of the PE technique and the cross-range wave number spectrum. ${ }^{3}$ The cross-range transform reduces the three-dimensional problem to a two-dimensional problem that can be solved using the existing two-dimensional PE models. However, the spectral PE solution breaks down for energy propagating nearly parallel to the cross-range direction. For three-dimensional scattering problems in a cylindrical coordinate system, a few PE models were developed by solving a PE using the finite difference method ${ }^{4}$ and using the alternating direction method. ${ }^{5}$ A coupled mode PE method including mode coupling terms is presented for large-scale scattering problems involving coupling of energy between modes and azimuths. ${ }^{6}$ An analytical solution for the three-dimensional acoustic scattering from a nonpenetrable cylindrical island in shallow water is

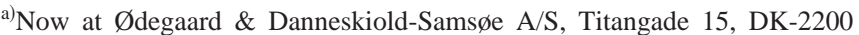
Copenhagen N, Denmark.
}

derived using a normal-mode, double-series expansion. ${ }^{7}$ The analytical solution is numerically efficient for relatively low frequencies. However, the models above for scattering problems in a cylindrical coordinate system were one-way models (i.e., for forward propagation only). In active sonar simulations where signals are transmitted and received at the same point, it is better to use a cylindrical coordinate system rather than a Cartesian coordinate system. Although a hybrid three-dimensional, two-way PE model ${ }^{8}$ may be applicable to model three-dimensional backscattering in a cylindrical coordinate system in some cases, it cannot handle fully threedimensional effects. Therefore, a PE model that can solve fully three-dimensional backscattering in the cylindrical coordinate system is required, and is presented in this article.

The three-dimensional, two-way PE model is mainly based on a radius-marching algorithm and a threedimensional, single scattering approach. The radiusmarching algorithm originates from a three-dimensional, one-way PE model $^{4}$ that achieves a wide-angle capability using a quadratic operator approximation. ${ }^{4}$ The threedimensional, single scattering approach arises from the idea introduced in a two-dimensional, two-way PE model. ${ }^{9}$ The three-dimensional computing domain is discretized as a sequence of stepwise radius-independent regions. The radiusmarching algorithm marches a wave field forward and backward in the radial direction within each region. Based on the continuity conditions of acoustic pressure and normal particle velocity across the vertical interface between regions, a specific boundary equation is established to obtain the backscattered field. For simplicity of the solution technique, a linear operator approximation is presently used to form the specific boundary equation. In future works other wide-angle approximations may be implemented to improve the model.

The outgoing wave equation is essentially based on a paraxial approximation, a far-field approximation and an as- 


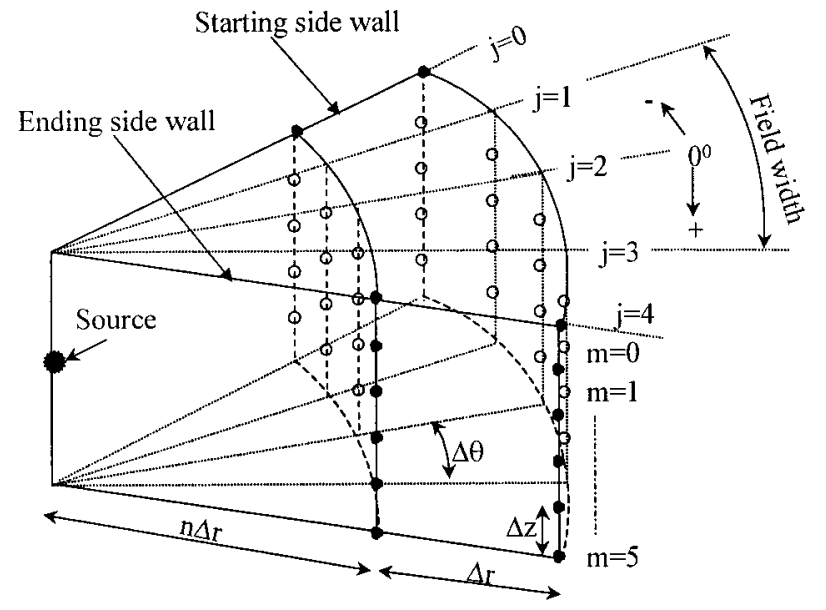

FIG. 1. The three-dimensional grid mesh for $J=3$ and $M=4$.

sumption that backscattering is negligible. ${ }^{1}$ The paraxial approximation limits sound propagation in the directions having small angles with respect to the horizontal. However, this narrow-angle limitation has been relaxed nowadays using a high-order Padé approximation. ${ }^{9}$ The far-field assumption (i.e., $k_{0} r \gg 0$ ) causes the solution to be valid only beyond a certain distance from the source. The third assumption is not needed in the two-way PE where backscattering has to be included. As the solution of outgoing and incoming waves can be expressed as combinations of Hankel functions, ${ }^{12}$ the solution breaks down at the source position because of the singularity of the Hankel functions. Should the solution be regular at the source position, the incident field had to be modified by the presence of the scatterer so that a part of the outgoing field combined with the incoming field could produce a regular result. This is, in fact, a multiple scattering effect that is beyond the scope of this article. Nevertheless, the use of the far-field assumption has implied that the valid solution domain is defined in such a way that a small neighborhood of the source is excluded. Consequently, the solution is not singular within the valid solution domain.

Different from the two-dimensional modeling, the threedimensional computations in the cylindrical coordinate system involve two additional sidewall boundaries, i.e., a starting sidewall and an ending sidewall. The starting sidewall refers to the radius-depth plane at the starting azimuth angle of the computing domain. The ending sidewall means the radius-depth plane at the ending azimuth angle of the computing domain. If computations are performed in a 360degree sector (i.e., a cylinder space), the physically correct sidewall boundary conditions are periodic because the starting sidewall overlaps the ending sidewall, while if a scattering object locates within a very narrow sector, the computing domain may be truncated into a sector less than 360 degrees. As a result, the computational time can be reduced greatly. However, this type of computation requires absorbing sidewall boundary conditions that are very difficult to implement. Therefore, approximate sidewall boundary conditions are presently used. The absorbing sidewall boundary conditions may be implemented in further works.

There is a major drawback of using the cylindrical coordinate system to model three-dimensional backscattering, which will be discussed later in this article. Because the radius-marching algorithm always marches the wave field back to the source, the backscattered energy propagating in other directions may not be fully handled. The backscattering solution is thus valid within a limited area. This drawback is analyzed and explained using a geometric-optical interpretation.

It is clear that the single scattering approach is valid for problems involving a single interface, ${ }^{9}$ but it is not clear how well this approach works for problems involving multiple interfaces. Therefore, the present computations are focused on the backscattering from those objects that have a single cylindrical vertical surface. Some interesting threedimensional backscattering phenomena are also discussed.

\section{THE THREE-DIMENSIONAL, RADIUS-MARCHING ALGORITHM}

The derivation is performed in a cylindrical coordinate system $(r, \theta, z)$ and begins with the outgoing wave equation $(+)$ and the incoming wave equation $(-)$ :

$$
\begin{aligned}
& \frac{\partial}{\partial r} u^{+}=i k_{0}(Q-1) u^{+}, \\
& \frac{\partial}{\partial r} u^{-}=-i k_{0}(Q-1) u^{-},
\end{aligned}
$$

where $k_{0}$ is a reference wave number. The wave fields $u^{+}$ and $u^{-}$are related to the acoustic pressures $p^{+}$and $p^{-}$via the Hankel functions, $\mathrm{H}_{0}^{(1)}$ and $\mathrm{H}_{0}^{(2)}$, which are given in the forms of their far-field asymptotic expressions $\left(k_{0} r \gg 1\right)$ :

$$
\begin{aligned}
p^{+}(r, \theta, z) & =u^{+}(r, \theta, z) \mathrm{H}_{0}^{(1)}\left(k_{0} r\right) \\
& \approx u^{+}(r, \theta, z) \sqrt{\frac{2}{\pi k_{0} r}} e^{i k_{0} r-i \pi / 4}, \\
p^{-}(r, \theta, z) & =u^{-}(r, \theta, z) \mathrm{H}_{0}^{(2)}\left(k_{0} r\right) \\
& \approx u^{-}(r, \theta, z) \sqrt{\frac{2}{\pi k_{0} r}} e^{-i k_{0} r+i \pi / 4} .
\end{aligned}
$$

Note that the solutions of Eq. (2a) and Eq. (2b) are not singular in the far field (i.e., $r \gg 1 / k_{0}$ ). The three-dimensional operator $Q \equiv \sqrt{1+X+Y}$ is defined by

$$
\begin{aligned}
& X=\xi^{2}(r, \theta, z)-1+\frac{1}{k_{0}^{2}} \rho \frac{\partial}{\partial z}\left(\frac{1}{\rho} \frac{\partial}{\partial z}\right), \\
& Y=\frac{1}{k_{0}^{2} r^{2}} \rho \frac{\partial}{\partial \theta}\left(\frac{1}{\rho} \frac{\partial}{\partial \theta}\right),
\end{aligned}
$$

where $\xi=c_{0} / c(r, \theta, z)$ represents the index of refraction, $c_{0}$ is a reference sound speed, and $\rho=\rho(\theta, z)$ denotes the density of the medium. Since $Y$ is proportional to $1 / r^{2}$, the azimuthal coupling in relation to $Y$ reduces quickly when the wave field is marched out in radius. At very large radii, the azimuthal coupling becomes unimportant.

In a cylindrical coordinate system, a three-dimensional environment is discretized in the $z$ direction, $r$ direction, and $\theta$ direction. A general three-dimensional grid mesh is shown in Fig. 1, where the computing domain is a sector. The steps 


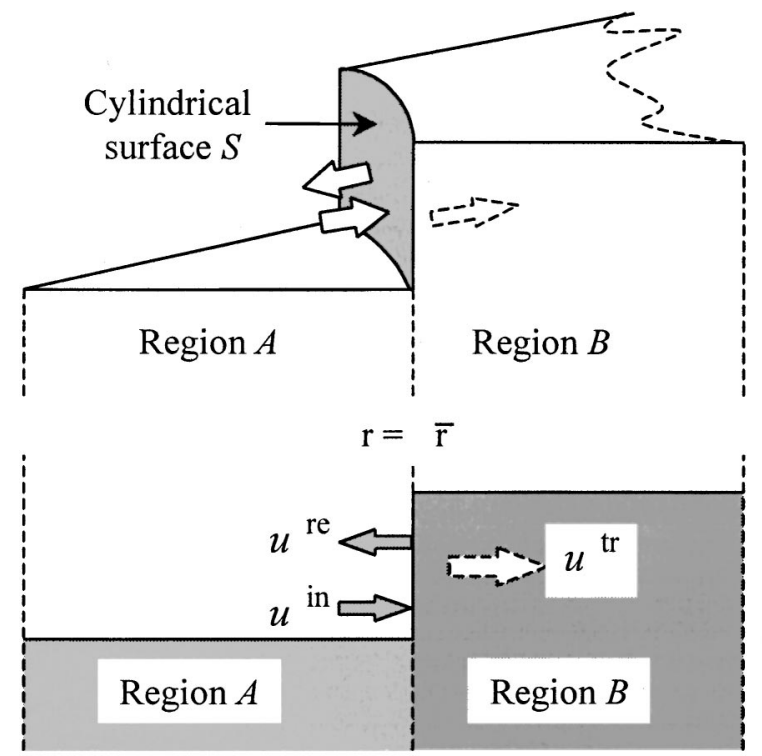

(a)

(b)

FIG. 2. A three-dimensional view (a) and a side view (b) of the geometry of a step object.

in range, in azimuth, and in depth are denoted by $\Delta r, \Delta \theta$, and $\Delta z$, respectively. The range index is denoted by $n$. The sector boundaries are identified by $j=1-J(J=3$ in the example in Fig. 1). In particular, the starting sidewall boundary is denoted by $j=0$, and the ending sidewall boundary is denoted by $j=J+1$. The depth index is denoted by $m=1-M$ $(M=4$ in Fig. 1). The sea surface is indicated by $m=0$, and the horizontal interface at the maximum depth is indicated by $m=M+1$. Thus, $u_{m j}^{n}$ denotes the value of a point at the position of $r=n \Delta r, \theta=j \Delta \theta$, and $z=m \Delta z$.

A range-dependent environment, where the waterseabed interface may vary in the radial direction as well as in the azimuthal direction, is discretized forming a sequence of stepwise, range-independent regions shown in Fig. 2. Two regions $A$ and $B$ are separated by a cylindrical interface $(S)$ at $r=\bar{r}$. Sound is assumed to propagate from $A$ to $B$ across $S$. The incident field, the transmitted field, and the reflected field are denoted by $u^{\mathrm{in}}, u^{\mathrm{tr}}$, and $u^{\mathrm{re}}$, respectively. Note that the azimuthal discretization may cause discrepancies between the real (original) environment and the discrete environment. Analyses of the errors arising from the azimuthal discretization can be found in Ref. 8 .

The radius-marching algorithm is based on the quadratic approximation ${ }^{4,10}$

$$
Q \equiv \sqrt{1+X+Y} \cong 1+\frac{1}{2} X-\frac{1}{8} X^{2}+\frac{1}{2} Y .
$$

The radius-marching algorithm can be expressed as ${ }^{3}$

$$
\begin{aligned}
{[1+} & \left.\frac{1-i k_{0} \Delta r}{4} X\right]\left[1-\frac{i k_{0} \Delta r}{4} Y\right] u^{n+1} \\
& =\left[1+\frac{1+i k_{0} \Delta r}{4} X\right]\left[1+\frac{i k_{0} \Delta r}{4} Y\right] u^{n},
\end{aligned}
$$

where $u^{n}$ denotes the known wave field at the present range (range $n$ ), and $u^{n+1}$ denotes the unknown wave field at the subsequent range (range $n+1)$. Since in Eq. (5) the couplings between $X$ and $Y$ are separated, Eq. (5) can be solved efficiently by solving two tridiagonal system of equations using the lower-upper triangular (LU) decomposition method. ${ }^{11}$

\section{THE THREE-DIMENSIONAL, SINGLE SCATTERING APPROACH}

As shown in Fig. 2, across a cylindrical interface $S$ at $r=\bar{r}$, the acoustic pressure and the normal components of the particle velocity satisfy the continuity conditions:

$$
\begin{aligned}
& u^{\mathrm{in}} H_{0}^{(1)}\left(k_{0} \bar{r}\right)+u^{\mathrm{re}} H_{0}^{(2)}\left(k_{0} \bar{r}\right)=u^{\mathrm{tr}} H_{0}^{(1)}\left(k_{0} \bar{r}\right), \\
& \left.\frac{1}{\rho_{A}} \frac{\partial\left(u^{\mathrm{in}} H_{0}^{(1)}\right)}{\partial r}\right|_{\bar{r}}+\left.\frac{1}{\rho_{A}} \frac{\partial\left(u^{\mathrm{re}} H_{0}^{(2)}\right)}{\partial r}\right|_{\bar{r}}=\left.\frac{1}{\rho_{B}} \frac{\partial\left(u^{\mathrm{tr}} H_{0}^{(1)}\right)}{\partial r}\right|_{\bar{r}}
\end{aligned}
$$

Replacing the derivatives in Eq. (6b) using Eqs. (1a) and (1b), the combination of Eqs. (6a) and (6b) results in a threedimensional boundary equation:

$$
\left[L_{A}+L_{B}-\frac{i}{2 k_{0} \bar{r}}\left(\frac{1}{\rho_{A}}-\frac{1}{\rho_{B}}\right)\right] u^{\mathrm{tr}}=2 L_{A} u^{\mathrm{in}},
$$

where

$$
\begin{gathered}
L_{A}=\frac{\sqrt{1+X_{A}+Y_{A}}}{\rho_{A}} \approx \frac{1+0.5 X_{A}+0.5 Y_{A}}{\rho_{A}}, \\
L_{B}=\frac{\sqrt{1+X_{B}+Y_{B}}}{\rho_{B}} \approx \frac{1+0.5 X_{B}+0.5 Y_{B}}{\rho_{B}} .
\end{gathered}
$$

A low-order expansion is used here to approximate the square-root operator in Eq. (8). Although this is a narrowangle approximation for propagation in azimuth-depth plane, it gives the simplest finite difference scheme to the operator $L$. Other approximations such as a rational-function approximation may be used; however, discretizing Eq. (7) will be much more difficult and complicated because operators are involved in both the numerator and denominator. Once $u^{\text {tr }}$ is obtained, the reflected field $u^{\text {re }}$ can be readily found:

$$
u^{\mathrm{re}}=-i \cdot e^{i 2 k_{0} \bar{r}}\left(u^{\mathrm{tr}}-u^{\mathrm{in}}\right) .
$$

The continuity conditions in the radial direction are fulfilled by satisfying Eq. (7), while the continuity conditions in the azimuthal direction are fulfilled automatically during the radius-marching process that includes the azimuthal coupling and azimuthal dependence.

\section{THE SIDEWALL BOUNDARY CONDITIONS}

Solving Eq. (7) forms the crucial part in the development of the present model. The solution technique greatly depends on how the operator $L$ is approximated. Since Eq. (8) only involves $X$ and $Y$ in the numerator, the operator $L$ can be discretized using the finite difference scheme and Varga's procedure ${ }^{10}$ that satisfies the azimuthal continuity. Using the notations shown in Fig. 1, the operation $L u$ can be expressed as

$$
\begin{aligned}
L u_{m, j} \equiv & B_{m, j} u_{m, j}+D_{m, j} u_{m+1, j}+E_{m, j} u_{m-1, j}+F_{m, j} u_{m, j+1} \\
& +G_{m, j} u_{m, j-1},
\end{aligned}
$$


where $B_{m, j}, D_{m, j}, E_{m, j}, F_{m, j}$, and $G_{m, j}$ are the constant coefficients given in the Appendix. Unlike Eq. (5), Eq. (7) is solved at one range only. Therefore, the superscript $n$ is omitted in Eq. (10).

To rewrite Eq. (10) in the form of a product of matrices, proper boundary conditions for the starting sidewall and the ending sidewall are needed. Two types of sidewall boundary conditions are implemented corresponding to whether the computing domain is 360 degrees or less than 360 degrees in azimuth. To clearly illustrate how these sidewall boundary conditions are implemented, a simple example given in Fig. 3 is used.

In general the computing domain is discretized as sectors shown in Fig. 3(a). The sector boundaries are counted from the starting sidewall $(j=0)$ to the ending sidewall $(j$ $=4)$. Imagine to rotate the ending sidewall $(j=4)$ clockwise and the starting sidewall $(j=0)$ counter-clockwise until one sector is overlapped, the computing domain becomes a circular area shown in Fig. 3(b). The sector boundaries are numbered from $j=0$ to $j=4$ in the direction shown by the arrows. The periodic sidewall boundary conditions applied to Fig. 3(b) assume that $u_{m, 0}=u_{m, 3}$ and $u_{m, 4}=u_{m, 1}$ because one sector is overlapped, shown by the dark shadow sector. Using these assumptions, Eq. (10) can be rewritten as

$$
\begin{aligned}
\mathbf{L} \cdot \mathbf{U}= & {\left[\begin{array}{ccccccc}
\mathbf{T}_{1} & \mathbf{F}_{1} & 0 & \cdots & \cdots & 0 & \mathbf{G}_{1} \\
\mathbf{G}_{2} & \mathbf{T}_{2} & \mathbf{F}_{2} & \ddots & & & 0 \\
0 & \mathbf{G}_{3} & \mathbf{T}_{3} & \ddots & \ddots & & \vdots \\
\vdots & \ddots & \ddots & \ddots & \ddots & \ddots & \vdots \\
\vdots & & \ddots & \ddots & \mathbf{T}_{J-2} & \mathbf{F}_{J-2} & 0 \\
0 & & & \ddots & \mathbf{G}_{J-1} & \mathbf{T}_{J-1} & \mathbf{F}_{J-1} \\
\mathbf{F}_{J} & 0 & \cdots & \cdots & 0 & \mathbf{G}_{J} & \mathbf{T}_{J}
\end{array}\right] } \\
& \cdot\left(\begin{array}{c}
\mathbf{u}_{1} \\
\mathbf{u}_{2} \\
\vdots \\
\vdots \\
\vdots \\
\mathbf{u}_{J-1} \\
\mathbf{u}_{J}
\end{array}\right)+\left(\begin{array}{c}
\mathbf{v}_{1} \\
\mathbf{v}_{2} \\
\vdots \\
\vdots \\
\vdots \\
\mathbf{v}_{J-1} \\
\mathbf{v}_{J}
\end{array}\right),
\end{aligned}
$$

where the block matrices are defined by

$$
\begin{aligned}
\mathbf{T}_{i} & =\left[\begin{array}{cccc}
B_{1, i} & D_{1, i} & 0 & 0 \\
E_{2, i} & \ddots & \ddots & 0 \\
0 & \ddots & \ddots & D_{M-1, i} \\
0 & 0 & E_{M, i} & B_{M, i}
\end{array}\right], \\
\mathbf{F}_{i} & =\left[\begin{array}{cccc}
F_{1, i} & 0 & \cdots & 0 \\
0 & \ddots & \ddots & \vdots \\
\vdots & \ddots & \ddots & 0 \\
0 & \cdots & 0 & F_{M, i}
\end{array}\right]
\end{aligned}
$$

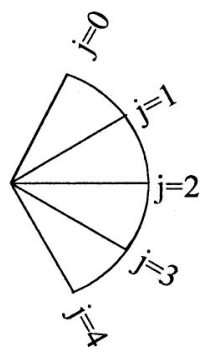

(a)

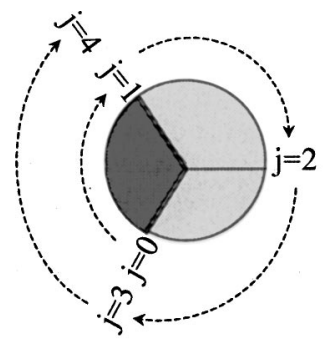

(b)

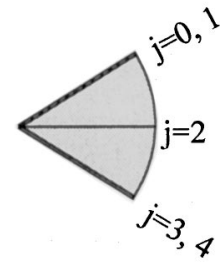

(c)
FIG. 3. Top view of sector boundaries. (a) General mesh. (b) Computing domain around 360 degrees. (c) Computing domain less than 360 degrees.

$$
\mathbf{G}_{i}=\left[\begin{array}{cccc}
G_{1, i} & 0 & \cdots & 0 \\
0 & \ddots & \ddots & \vdots \\
\vdots & \ddots & \ddots & 0 \\
0 & \cdots & 0 & G_{M, i}
\end{array}\right]
$$

Each $\mathbf{T}_{i}$ is a tridiagonal matrix, while $\mathbf{F}_{i}$ and $\mathbf{G}_{i}$ are diagonal matrices. The elements $B_{m, j}, D_{m, j}, E_{m, j}, F_{m, j}$, and $G_{m, j}$ are given in the Appendix. The vectors $\mathbf{u}_{i}$ and $\mathbf{v}_{i}$ contain the values of the field points at the $i$ th sector boundary. They are given by

$$
\mathbf{u}_{i}=\left(\begin{array}{c}
u_{1, i} \\
u_{2, i} \\
\vdots \\
u_{M-1, i} \\
u_{M, i}
\end{array}\right), \quad \mathbf{v}_{i}=\left(\begin{array}{c}
E_{1, i} \cdot u_{0, i} \\
0 \\
\vdots \\
0 \\
D_{M, i} \cdot u_{M+1, i}
\end{array}\right)
$$

where $u_{1, i}$ to $u_{M, i}$ represent the values of the field points in the $z$ direction, $u_{0, i}$ denotes the value on the sea surface, and $u_{M+1, i}$ denotes the value at the maximum depth. The vector $\mathbf{v}_{i}$ will vanish if a pressure-release boundary condition is assumed on the sea surface and at the maximum depth.

If the computing domain is a sector less than 360 degrees, absorbing boundary conditions should be applied to the sidewall boundaries to prevent artificial reflections. However, it is difficult to implement the absorbing boundary conditions. Presently, approximate sidewall boundary conditions shown in Fig. 3(c) are used, which set the starting sidewall $(j=0)$ the same as the sector boundary of $j=1$, and set the ending sidewall $(j=4)$ the same as the sector boundary of $j=3$, namely, $u_{m, 0}=u_{m, 1}$ and $u_{m, 4}=u_{m, 3}$. Based on the approximate sidewall boundary conditions, a new sparse matrix equation is derived from Eq. (10). Slightly different from Eq. (11a), the new sparse matrix does not involve the block matrices $\mathbf{G}_{1}$ and $\mathbf{F}_{J}$ at the corners. In addition, the new sparse matrix has two block matrices $\mathbf{T}_{1}$ and $\mathbf{T}_{J}$ defined differently from Eq. (11b). The new $\mathbf{T}_{1}$ and $\mathbf{T}_{J}$ are defined by

$$
\mathbf{T}_{1}=\left[\begin{array}{cccc}
B_{1,1}+G_{1,1} & D_{1,1} & 0 & 0 \\
E_{2,1} & \ddots & \ddots & 0 \\
0 & \ddots & \ddots & D_{M-1,1} \\
0 & 0 & E_{M, 1} & B_{M, 1}+G_{M, 1}
\end{array}\right],
$$




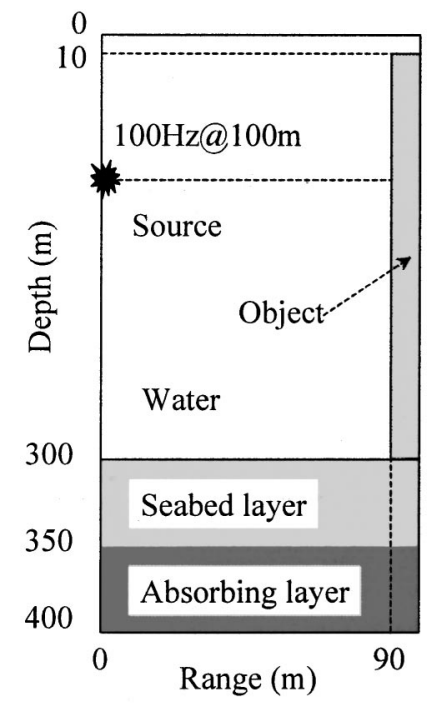

(a)

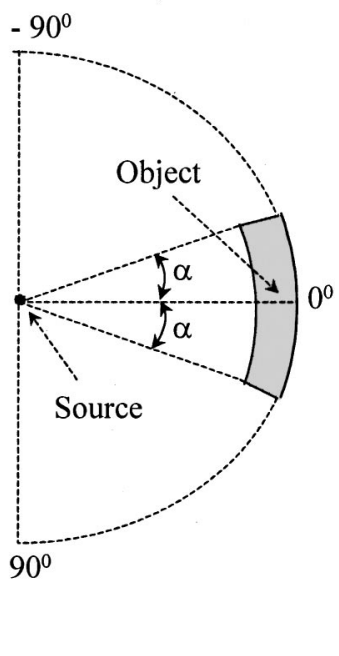

(b)
FIG. 4. The geometry of the single sector case. (a) The side view. (b) The top view.

$$
\mathbf{T}_{J}=\left[\begin{array}{cccc}
B_{1, J}+F_{1, J} & D_{1, J} & 0 & 0 \\
E_{2, J} & \ddots & \ddots & 0 \\
0 & \ddots & \ddots & D_{M-1, J} \\
0 & 0 & E_{M, J} & B_{M, J}+F_{M, J}
\end{array}\right]
$$

Note that only the main diagonals in $\mathbf{T}$ in Eq. (12) are different from Eq. (11b).

Using either of the sidewall boundary conditions, Eq. (7) can finally be expressed in the form of a sparse matrix equation. As shown in Eqs. (11a) and (11b), the size of the matrix $L$ is of the order of $M^{2} \times J^{2}$, where $M$ is the total number of points in the $z$ direction, and $J$ is the number of sectors in the $\theta$ direction. The matrix $L$ may be very huge but has only very few nonzero diagonals. In the case of Fig. 3(b), $L$ has $5 M J$ $-2 J$ nonzero elements. In the case of Fig. 3(b), $L$ has only $5 M J-2(M+J)$ nonzero elements. The sparse matrix equation is solved using a row-index method, ${ }^{11}$ which stores only nonzero elements during the computation.

\section{EXAMPLES}

An example shown in Fig. 4 is used to verify the two different sidewall boundary conditions discussed above. As shown in the side view of Fig. 4(a), the water is 300 deep with the sound speed $c_{w}=1500 \mathrm{~m} / \mathrm{s}$, density $\rho_{w}$ $=1000 \mathrm{~kg} / \mathrm{m}^{3}$, and attenuation $\alpha_{w}=0.0 \mathrm{~dB} / \lambda$. The $50-\mathrm{m}$ seabed layer is described by $c_{b}=1700 \mathrm{~m} / \mathrm{s}, \rho_{b}=1500 \mathrm{~kg} / \mathrm{m}^{3}$, and $\alpha_{b}=0.5 \mathrm{~dB} / \lambda$. The absorbing layer extended from 350 to $400 \mathrm{~m}$ has the same parameters as in the seabed layer except $\alpha_{b}=30.0 \mathrm{~dB} / \lambda$. An omnidirectional point source is assumed locating at $100 \mathrm{~m}$ below the sea surface and transmitting continuous wave signals with a frequency of $100 \mathrm{~Hz}$. A cylindrical sector is used as the scattering object because it has only one vertical cylindrical interface. To concentrate on the effects of backscattering from the object, the influence from the interaction of sound with the seabed should be reduced greatly. Therefore, the object is placed at the range of
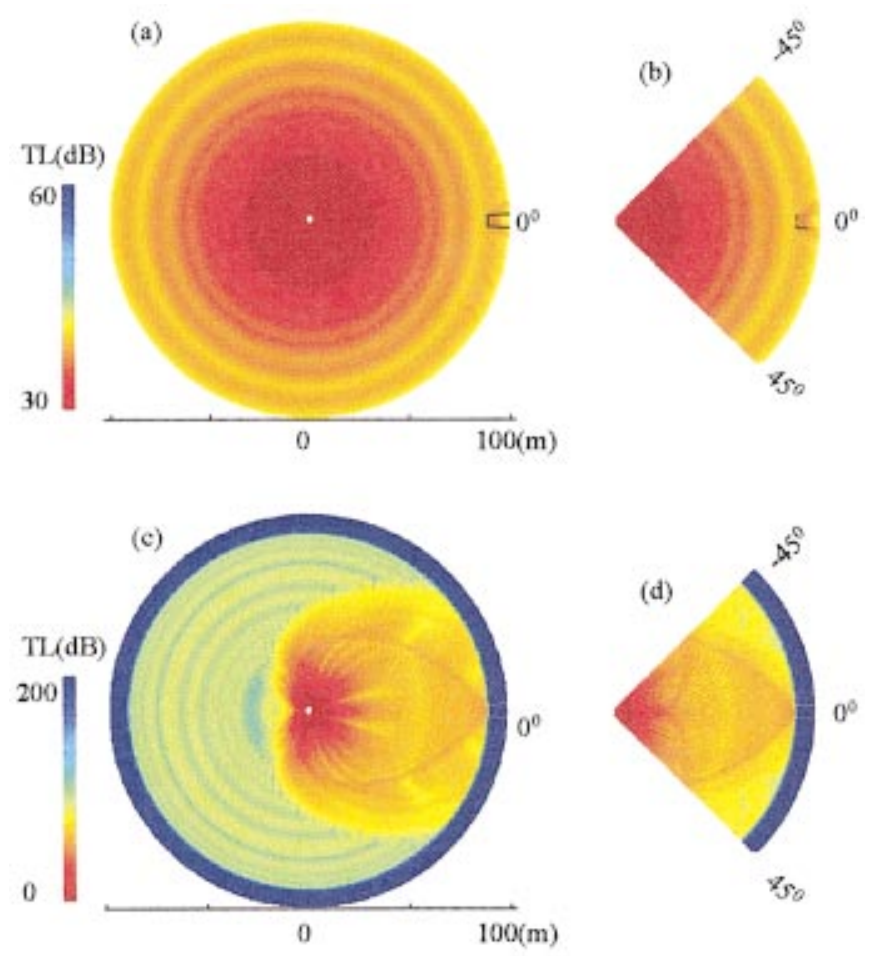

FIG. 5. The top views of the forward transmission losses, (a) and (b), and the backward transmission losses, (c) and (d). The computing domain is 360 degrees in (a) and (c), but 90 degrees in (b) and (d). All results are created at the receiving depth of $100 \mathrm{~m}$, and the same object within an azimuthal angle of 4 degrees is used.

$90 \mathrm{~m}$ that is much shorter than the water depth to reduce the bottom influence. The object is assumed situating from $-\alpha$ to $\alpha$ in the azimuthal direction shown in Fig. 4(b). In this example $\alpha$ equals 2 degrees. To use the two different sidewall boundary conditions, the computing domain is chosen to be a 360-degree sector in one test case and a 90-degree sector in another test case. The trial runs show that the parameters of $\Delta r=1 \mathrm{~m}, \Delta z=1 \mathrm{~m}$, and $\Delta \theta=1$ degree may be appropriate to obtain the convergent results.

The top views of the forward and backward transmission losses obtained at the receiving depth of $100 \mathrm{~m}$ are shown in Fig. 5, where the top plots display the forward transmission losses, and the lower plots are the backward transmission losses. The left plots correspond to the 360-degree computations, while the right plots show the results calculated in a sector area from -45 degrees to 45 degrees. The comparison between Figs. 5(a) and 5(b) and the comparison between Figs. 5(c) and 5(d) both display very good agreement in the scattering patterns. Significant diffraction effects are found in Figs. 5(c) and 5(d), where energy is backscattered from the object in a manner similar to sound radiation from a point source. Quantitative comparisons are given in Figs. 6 and 7. Figure 6 shows the transmission losses versus azimuthal angles at a given range. The plotting depth is $100 \mathrm{~m}$, and the plotting range is $100 \mathrm{~m}$ for the forward propagation and $70 \mathrm{~m}$ for the backward propagation. The solid curve corresponds to the computation from -180 degrees to 180 degrees, and the dashed curve corresponds to the computation from -45 degrees to 45 degrees. The forward and backward transmission losses created at the azimuthal angle of 0 degrees and at 

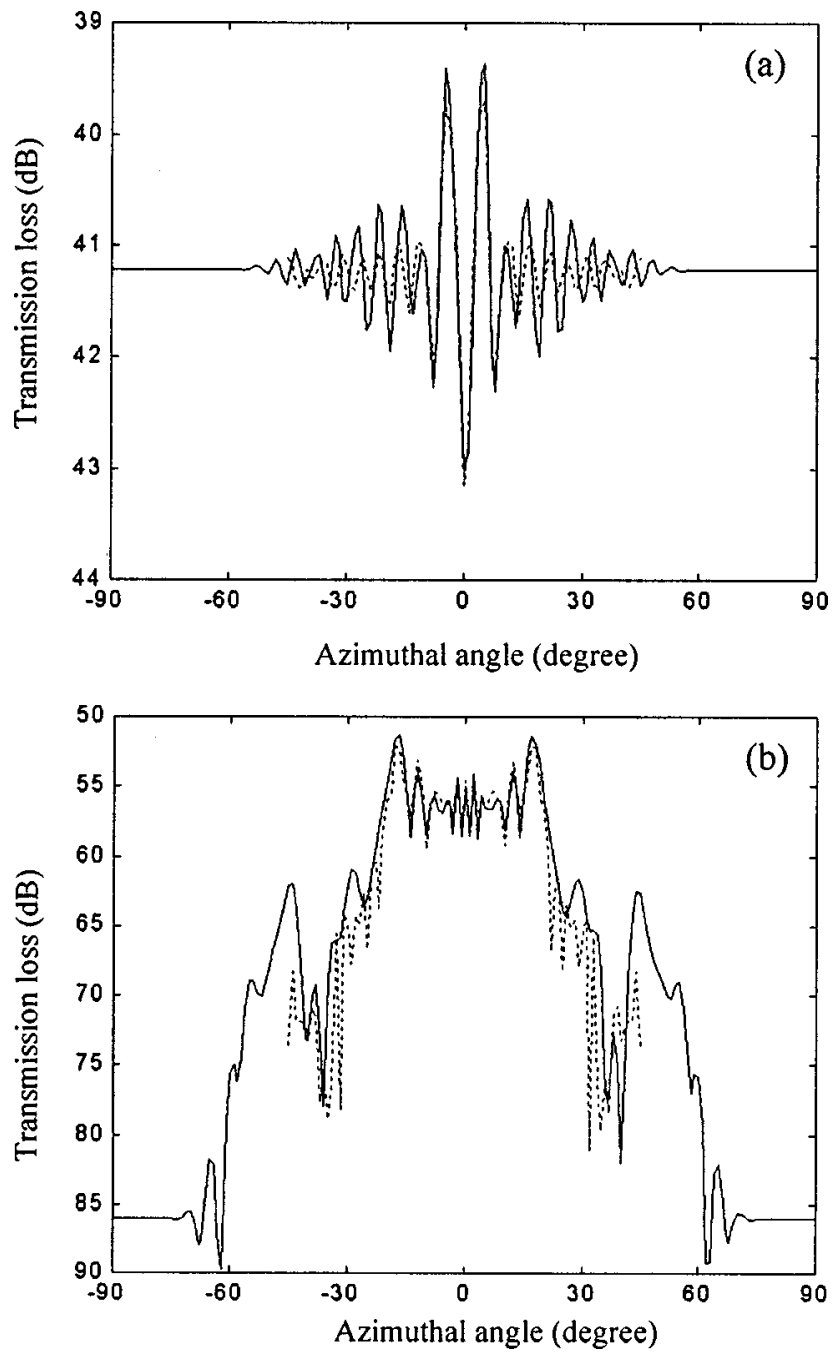

FIG. 6. Comparisons of the transmission losses for (a) the forward propagation plotted at the radius of $100 \mathrm{~m}$, and (b) the backward propagation plotted at the radius of $70 \mathrm{~m}$. The solid and dashed curves correspond to the computation over 360 degrees and the calculation within a 90-degree sector, respectively.

the depth of $100 \mathrm{~m}$ are shown in Fig. 7, where the solid curve corresponds to the 360-degree computation and the squares are the result of the 90-degree calculation. Excellent agreement is found in all the comparisons in Figs. 6 and 7, indicating that both of the sidewall boundary conditions shown in Fig. 3 can be applied to obtain identical results. However, the computational time due to the use of two different sidewall boundary conditions is very different. In the example above, the two-way run of the 360-degree computation takes about $10 \mathrm{~min}$ on a $400-\mathrm{MHz} \mathrm{PC}$, while the two-way run of the 90-degree calculation uses only about $1 \mathrm{~min}$ on the same computer. Therefore, it is not always preferable to perform 360-degree calculation using the periodic sidewall boundary condition if computations in a sector area less than 360 degrees can cover the backscattered field that is of interest, and the solution accuracy is acceptable.

The side views of the forward and backward transmission losses created at an azimuthal angle of 0 degrees for the 360-degree and the 90-degree computations are given in Fig. 8. Excellent agreement is observed in the comparison of the forward propagating field between Figs. 8(a) and 8(b), and in
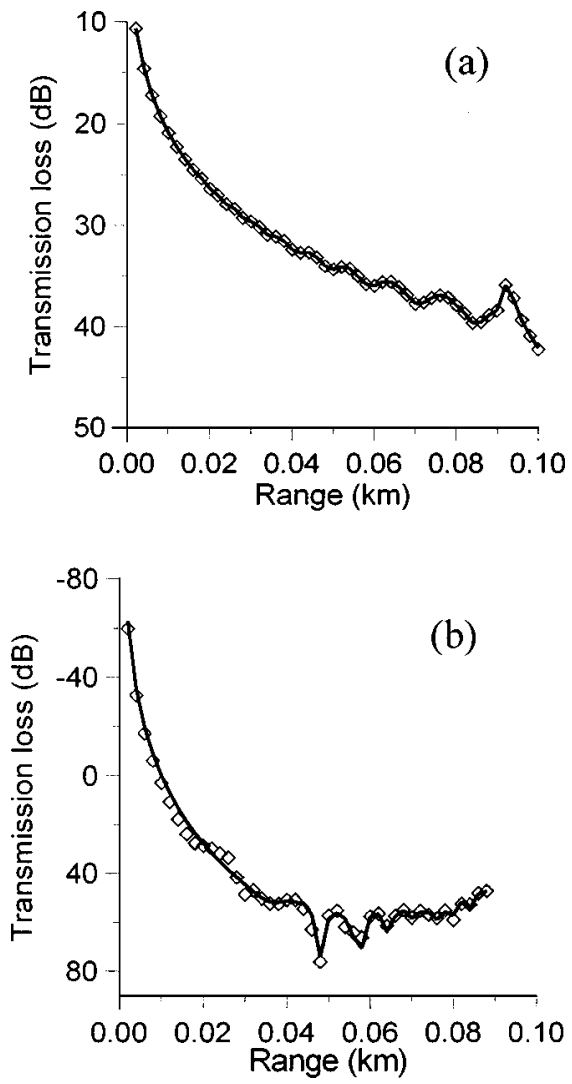

FIG. 7. The forward transmission loss (a) and the backward transmission loss (b) created at azimuthal angle of 0 degrees and at the depth of $100 \mathrm{~m}$. Comparisons between the solid curve for the 360-degree computation and the squares for the 90-degree calculation show an excellent agreement.

the comparison of the backward propagating field between Figs. 8(c) and 8(d). It is also noted that the absorbing layer is efficient enough to reduce the bottom reflection by attenuating significantly the sound energy that penetrates into the bottom. Therefore, the backscattered fields given in Fig. 5 are mostly due to the backscattering from the cylindrical sector object with minimized bottom influences. The results thus are more useful and accurate for studying the characteristics of the backscattering from objects.

Backscattering effects are of course dependent on the source frequency as well. To illustrate the frequency dependence, the source frequency is increased from 100 to $300 \mathrm{~Hz}$ in the previous example. The side views of the forwardpropagating field and the backward-propagating field created at azimuthal angle of 0 degrees are shown in Figs. 9(a) and 9(b). In Fig. 9(a) the major change from Fig. 8(a) is that the sound energy radiating from the point source is trapped within a beam, which ensonifies the object very well. Figure 9(b) shows significant energy being backscattered from the object, where the lower edge of the backscattered field is along the specular direction of the lower edge of the incident field. Bottom influence can be ignored in this case.

Figure 10 shows the top view of the backward transmission loss obtained at the receiving depth of $100 \mathrm{~m}$. The backscattering pattern shows a beam that is narrower than the one found in Fig. 5(c), meaning that the diffraction effects are weaker in Fig. 10. It is noted that the backscattered energy goes all the way back to the source. However, this is not 

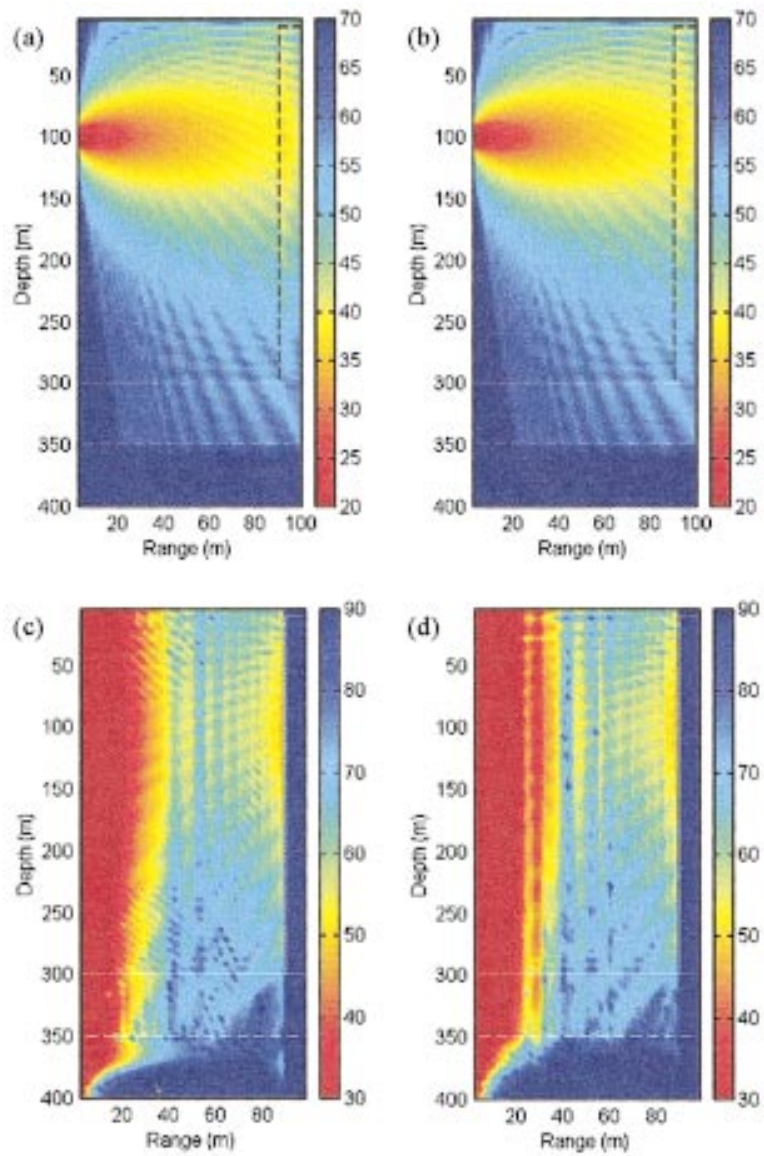

FIG. 8. The side views of the forward transmission losses, (a) and (b), and the backward transmission losses, (c) and (d), created at the azimuthal angle of 0 degrees. The plots to the left correspond to the 360-degree computations, and the plots to the right are the results of the 90-degree calculations. The horizontal white solid lines indicate the water-seabed interface, the horizontal white dashed lines show the absorbing layer interface, and the vertical black and white lines outline the cylindrical interface of the object.

what is exactly observed in practice. Therefore, the solution cannot be taken seriously inside some range around the source. This is also the main drawback of using a cylindrical coordinate system for three-dimensional, two-way propagation modeling. A geometrical-optical interpretation displayed
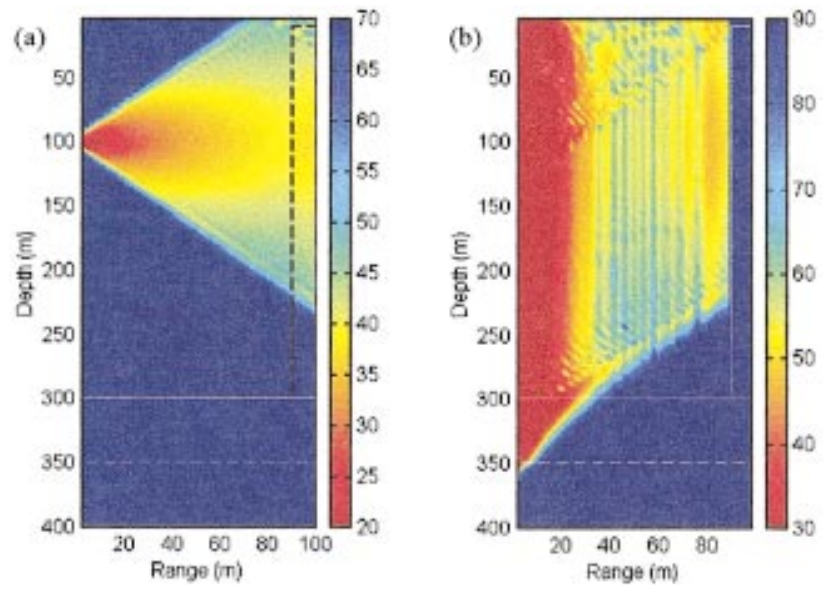

FIG. 9. The side views of the forward transmission loss (a) and the backward transmission loss (b) created at the azimuthal angle of 0 degrees for the frequency of $300 \mathrm{~Hz}$.

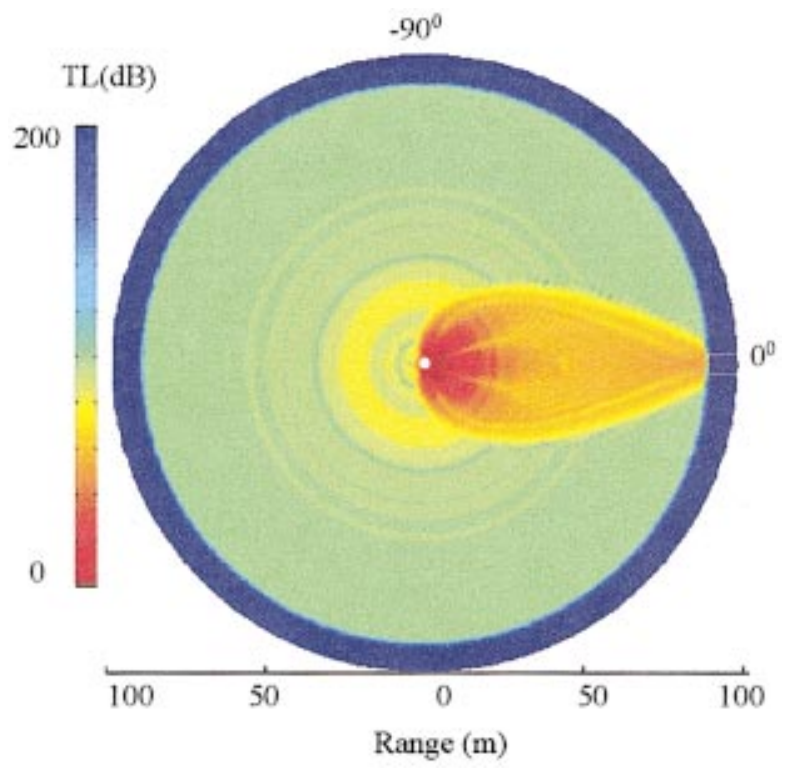

FIG. 10. The top view of the backward transmission loss created at the receiving depth of $100 \mathrm{~m}$. The source frequency is $300 \mathrm{~Hz}$.

in Fig. 11 may be helpful to roughly understand the phenomenon. The scatterer is assumed to be a very narrow object $(S)$ behaving like an omnidirectional point source that can radiate sound in all directions. The wave field is marched back to the source $(O)$ through the radii indicated by the dotted circles. An incoming propagation is defined as the sound propagation from large radii to small radii. For example, ray $S A$ is considered as an incoming propagation, while ray $A B$ is considered as an outgoing propagation. Since the backward radius-marching algorithm handles only the incoming propagation, the outgoing propagation is omitted during the backward marching process. Therefore, all the possible diffracting rays (indicated by the arrows) from the scatterer $S$ are "truncated" with only the incoming propagation parts

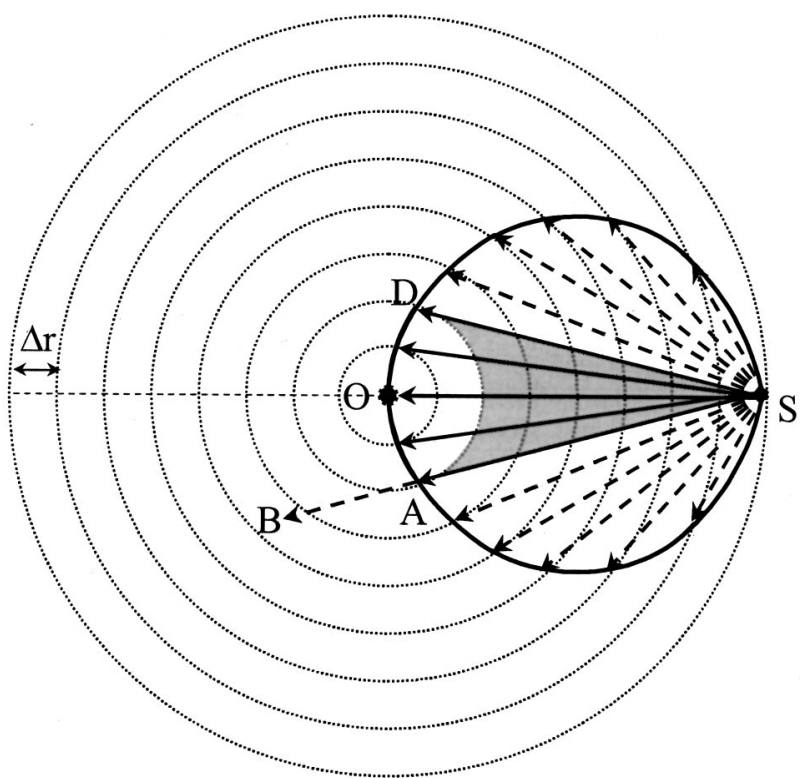

FIG. 11. A geometric-optical interpretation of the limited region of backscattering from a point scatterer. 
remaining during the backward marching. Those incoming rays representing all the valid solutions are bounded within a limited region shown by the thick elliptic curves. Furthermore, if the scatterer $S$ is not an omnidirectional point source but has certain directivity, the possible diffracting rays (now indicated by the solid arrows only) from $S$ are limited within a certain angle, which may be due to the PE's angular limitation. The far-field assumption also leads to a bound at a certain radius. The valid backscattering solutions represented by those solid arrows finally are bounded within a small area indicated by the shadow zone in Fig. 11, which is similar to the backscattering pattern in Fig. 10.

\section{DISCUSSIONS AND CONCLUSIONS}

A new three-dimensional, two-way PE model has been developed in a cylindrical coordinate system to solve threedimensional backscattering problems including azimuthal diffraction effects. The correct periodic sidewall boundary conditions are implemented for computations in a sector around 360 degrees. When the computing domain is less than 360 degrees, approximate sidewall boundary conditions are applied to give fairly good results. The accurate absorbing sidewall boundary conditions may be implemented in future works. Although the Hankel function is singular at the source position, the solution does not have a singularity because the use of the far-field assumption removes a small neighborhood of the source from the valid solution domain. The major drawback is that the backscattering solution is valid within a limited area, which is due to the cylindrical marching process as well as the single scattering approach.

Nevertheless, the three-dimensional, two-way PE model can be useful to demonstrate some interesting threedimensional backscattering phenomena that cannot be seen using two-dimensional models. For example, the results show that the backscattering from a narrow-angle object is in a manner similar to sound radiation from a point source. The azimuthal diffraction effects at higher frequencies are weaker than that at lower frequencies.

\section{ACKNOWLEDGMENTS}

The work was partly financed by the EU/MAST projects POSEIDON (MAS3-CT95-0038), LOTUS (MAS3-CT970099), and SIGMA (MAS3-CT97-0100).

\section{APPENDIX: DERIVATION OF THE SPARSE MATRIX EQUATION}

With the operator $L$ defined in Eq. (8), the operation $L u$ can be expressed as

$$
L u=\left[\frac{A_{0}+A_{1} X+A_{2} Y}{\rho}\right] u \equiv\left[L_{1}+L_{2}+L_{3}\right] u,
$$

where $A_{0}=1, A_{1}=A_{2}=0.5$. Inserting Eq. (3) into Eq. (A1) leads to

$$
\begin{aligned}
& L_{1}=A_{0} \frac{1}{\rho}+A_{1} \frac{\xi^{2}(r, \theta, z)-1}{\rho}, \quad L_{2}=\frac{A_{1}}{k_{0}^{2}} \frac{\partial}{\partial z}\left(\frac{1}{\rho} \frac{\partial}{\partial z}\right), \\
& L_{3}=\frac{A_{2}}{\left(k_{0} \bar{r}\right)^{2}} \frac{\partial}{\partial \theta}\left(\frac{1}{\rho} \frac{\partial}{\partial \theta}\right) .
\end{aligned}
$$

The varying density comprises the inhomogeneity of the medium. The derivation uses the notations shown in Fig. 1. Using the continuity conditions on the $m$ th horizontal interface $\left(z_{m}=z_{0}+m \Delta z\right)$ and the finite difference scheme, $L_{1}$ can be expressed as

$$
\begin{aligned}
L_{1} u_{m, j}= & \frac{A_{0}}{2}\left(\frac{1}{\rho_{m+1 / 2, j}}+\frac{1}{\rho_{m-1 / 2, j}}\right) u_{m, j} \\
& +\frac{A_{1}}{2}\left[\frac{\xi_{m+1 / 2, j}^{2}-1}{\rho_{m+1 / 2, j}}+\frac{\xi_{m-1 / 2, j}^{2}-1}{\rho_{m-1 / 2, j}}\right] u_{m, j} .
\end{aligned}
$$

For expressing $L_{2}$, the first-order derivative can easily be handled using a forward finite difference scheme. But the major concern is how to treat $(\partial / \partial z)((1 / \rho) \partial / \partial z)$ because $(1 / \rho) \partial / \partial z$ is discontinuous on the horizontal interface. Here, Varga's procedure ${ }^{10}$ is applied:

$$
\frac{\partial}{\partial z}\left(\frac{1}{\rho} \frac{\partial}{\partial z}\right) u_{m, j}=\frac{1}{\Delta z}\left[\left(\frac{1}{\rho} \frac{\partial}{\partial z}\right)_{m+1 / 2, j}-\left(\frac{1}{\rho} \frac{\partial}{\partial z}\right)_{m-1 / 2, j}\right] u_{m, j}=\frac{\frac{1}{\rho_{m+1 / 2, j}}\left(u_{m+1, j}-u_{m, j}^{1}\right)-\frac{1}{\rho_{m-1 / 2, j}}\left(u_{m, j}^{2}-u_{m-1, j}\right)}{(\Delta z)^{2}}
$$

where $u_{m, j}^{1}$ denotes the field on the horizontal interface $z_{m}$ in medium 1 , and $u_{m, j}^{2}$ denotes the field on the horizontal interface $z_{m}$ in medium 2 . The continuity conditions imply $u_{m, j}^{1}$ $=u_{m, j}^{2}=u_{m, j}$. Using the scheme in Eq. (A4), $L_{2}$ and $L_{3}$ can be expressed as

$$
\begin{aligned}
L_{2} u_{m, j}= & \frac{A_{1}}{\left(k_{0} \Delta z\right)^{2}}\left[-\left(\frac{1}{\rho_{m+1 / 2, j}}+\frac{1}{\rho_{m-1 / 2, j}}\right) u_{m, j}\right. \\
& \left.+\frac{1}{\rho_{m+1 / 2, j}} u_{m+1, j}+\frac{1}{\rho_{m-1 / 2, j}} u_{m-1, j}\right],
\end{aligned}
$$

$$
\begin{aligned}
L_{3} u_{m, j}= & \frac{A_{2}}{\left(k_{0} \bar{r} \Delta \theta\right)^{2}}\left[-\left(\frac{1}{\rho_{m, j+1 / 2}}+\frac{1}{\rho_{m, j-1 / 2}}\right) u_{m, j}\right. \\
& \left.+\frac{1}{\rho_{m, j+1 / 2}} u_{m, j+1}+\frac{1}{\rho_{m, j-1 / 2}} u_{m, j-1}\right] .
\end{aligned}
$$

Note that the densities in Eqs. (A3) and (A5) are evaluated at the depths of $z_{0}+\left(m+\frac{1}{2}\right) \Delta z$ and $z_{0}+\left(m-\frac{1}{2}\right) \Delta z$ on both sides of the $m$ th horizontal interface while the densities in Eq. (A6) are evaluated at the azimuthal angles of $\left(j+\frac{1}{2}\right) \Delta \theta$ and $\left(j-\frac{1}{2}\right) \Delta \theta$ on both sides of the $j$ th vertical interface. In- 
serting Eqs. (A3), (A5), and (A6) into (A1) results in Eq. (10), where the coefficients are given by

$$
\begin{aligned}
B_{m, j}= & \left(\frac{A_{0}}{2}-\frac{A_{1}}{\left(k_{0} \Delta z\right)^{2}}\right)\left(\frac{1}{\rho_{m+1 / 2, j}}+\frac{1}{\rho_{m-1 / 2, j}}\right) \\
& +\frac{A_{1}}{2}\left[\frac{\xi_{m+1 / 2, j}^{2}-1}{\rho_{m+1 / 2, j}}+\frac{\xi_{m-1 / 2, j}^{2}-1}{\rho_{m-1 / 2, j}}\right] \\
& -\frac{A_{2}}{\left(k_{0} \bar{r} \Delta \theta\right)^{2}}\left(\frac{1}{\rho_{m, j}+1 / 2}+\frac{1}{\rho_{m, j-1 / 2}}\right), \\
D_{m, j}= & \frac{A_{1}}{\left(k_{0} \Delta z\right)^{2}} \frac{1}{\rho_{m+1 / 2, j}}, \quad E_{m, j}=\frac{A_{1}}{\left(k_{0} \Delta z\right)^{2}} \frac{1}{\rho_{m-1 / 2, j}}, \\
F_{m, j}= & \frac{A_{2}}{\left(k_{0} \bar{r} \Delta \theta\right)^{2}} \frac{1}{\rho_{m, j+1 / 2}}, \quad G_{m, j}=\frac{A_{2}}{\left(k_{0} \bar{r} \Delta \theta\right)^{2}} \frac{1}{\rho_{m, j-1 / 2}} .
\end{aligned}
$$

For transforming Eq. (10) into a matrix form, a simple example shown in Fig. 3 is used, where $m=0-5$ and $j$ $=0-4$. Following the order of $j$ and $m$, Eq. (10) can be expanded as

$$
L u_{m, j}=\left\{\begin{array}{l}
B_{1,1} \cdot u_{1,1}+D_{1,1} u_{2,1}+E_{1,1} u_{0,1}+F_{1,1} u_{1,2}+G_{1,1} u_{1,0} \\
B_{2,1} u_{2,1}+D_{2,1} u_{3,1}+E_{2,1} u_{1,1}+F_{2,1} u_{2,2}+G_{2,1} u_{2,0} \\
B_{3,1} u_{3,1}+D_{3,1} u_{4,1}+E_{3,1} u_{2,1}+F_{3,1} u_{3,2}+G_{3,1} u_{3,0} \\
B_{4,1} u_{4,1}+D_{4,1} u_{5,1}+E_{4,1} u_{3,1}+F_{4,1} u_{4,2}+G_{4,1} u_{4,0} \\
B_{1,2} \cdot u_{1,2}+D_{1,2} u_{2,2}+E_{1,2} u_{0,2}+F_{1,2} u_{1,3}+G_{1,2} u_{1,1} \\
B_{2,2} u_{2,2}+D_{2,2} u_{3,2}+E_{2,2} u_{1,2}+F_{2,2} u_{2,3}+G_{2,2} u_{2,1} \\
B_{3,2} u_{3,2}+D_{3,2} u_{4,2}+E_{3,2} u_{2,2}+F_{3,2} u_{3,3}+G_{3,2} u_{3,1} \\
B_{4,2} u_{4,2}+D_{4,2} u_{5,2}+E_{4,2} u_{3,2}+F_{4,2} u_{4,3}+G_{4,2} u_{4,1} \\
B_{1,3} \cdot u_{1,3}+D_{1,3} u_{2,3}+E_{1,3} u_{0,3}+F_{1,3} u_{1,4}+G_{1,3} u_{1,2} \\
B_{2,3} u_{2,3}+D_{2,3} u_{3,3}+E_{2,3} u_{1,3}+F_{2,3} u_{2,4}+G_{2,3} u_{2,2} \\
B_{3,3} u_{3,3}+D_{3,3} u_{4,3}+E_{3,3} u_{2,3}+F_{3,3} u_{3,4}+G_{3,3} u_{3,2} \\
B_{4,3} u_{4,3}+D_{4,3} u_{5,3}+E_{4,3} u_{3,3}+F_{4,3} u_{4,4}+G_{4,3} u_{4,2} .
\end{array}\right.
$$

Equation (A10) may be written in the form of a product of matrices if $u$ is written as a vector following the order of $j$. However, of special concern is how to treat the starting sidewall boundary $\left(u_{m, 0}\right)$ and the ending sidewall boundary $\left(u_{m, 4}\right)$. Two types of sidewall boundary conditions are shown in Fig. 3.

In the case of Fig. 3(b), the periodic sidewall boundary conditions assume $u_{m, 0}=u_{m, 3}$ and $u_{m, 4}=u_{m, 1}$. Equation (A10) can be reorganized and expressed in the form of a sparse matrix:

$$
L u=\left[\begin{array}{cccc:cccc:cccc}
B_{1,1} & D_{1,1} & 0 & 0 & F_{1,1} & 0 & 0 & 0 & G_{1,1} & 0 & 0 & 0 \\
E_{2,1} & B_{2,1} & D_{2,1} & 0 & 0 & F_{2,1} & 0 & 0 & 0 & G_{2,1} & 0 & 0 \\
0 & E_{3,1} & B_{3,1} & D_{3,1} & 0 & 0 & F_{3,1} & 0 & 0 & 0 & G_{3,1} & 0 \\
0 & 0 & E_{4,1} & B_{4,1} & 0 & 0 & 0 & F_{4,1} & 0 & 0 & 0 & G_{4,1} \\
\hdashline G_{1,2} & 0 & 0 & 0 & B_{1,2} & D_{1,2} & 0 & 0 & F_{1,2} & 0 & 0 & 0 \\
0 & G_{2,2} & 0 & 0 & E_{2,2} & B_{2,2} & D_{2,2} & 0 & 0 & F_{2,2} & 0 & 0 \\
0 & 0 & G_{3,2} & 0 & 0 & E_{3,2} & B_{3,2} & D_{3,2} & 0 & 0 & F_{3,2} & 0 \\
0 & 0 & 0 & G_{4,2} & 0 & 0 & E_{4,2} & B_{4,2} & 0 & 0 & 0 & F_{4,2} \\
\hdashline F_{1,3} & 0 & 0 & 0 & G_{1,3} & 0 & 0 & 0 & B_{1,3} & D_{1,3} & 0 & 0 \\
0 & F_{2,3} & 0 & 0 & 0 & G_{2,3} & 0 & 0 & E_{2,3} & B_{2,3} & D_{2,3} & 0 \\
0 & 0 & F_{3,3} & 0 & 0 & 0 & G_{3,3} & 0 & 0 & E_{3,3} & B_{3,3} & D_{3,3} \\
0 & 0 & 0 & F_{4,3} & 0 & 0 & 0 & G_{4,3} & 0 & 0 & E_{4,3} & B_{4,3}
\end{array}\right]\left(\begin{array}{c}
u_{1,1} \\
u_{2,1} \\
u_{3,1} \\
u_{4,1} \\
-u_{1,2} \\
u_{2,2} \\
u_{3,2} \\
u_{4,2} \\
u_{1,3} \\
u_{2,3} \\
u_{3,3} \\
u_{4,3}
\end{array}\right)+\left(\begin{array}{c}
E_{1,1} u_{0,1} \\
0 \\
0 \\
D_{4,1} u_{5,1} \\
\hdashline E_{1,2} u_{0,2} \\
0 \\
0 \\
D_{4,2} u_{5,2} \\
E_{1,3} u_{0,3} \\
0 \\
0 \\
0 \\
D_{4,3} u_{5,3}
\end{array}\right),
$$

where the values on the surface $\left(u_{0, j}\right)$ and the values at the maximum depth $\left(u_{5, j}\right)$ are extracted and written in a vector. Following the order of $j$, the matrix in Eq. (A11) may be divided into block matrices as indicated by the dashed lines. This sparse matrix has only seven diagonals and can be divided into $J^{2}$ block matrices (each block has $M^{2}$ elements). Two diagonal blocks $G_{m, 1}$ and $F_{m, 3}$ occurring at the corners are due to the use of the periodic sidewall boundary conditions. Equation (A11) can be written in the form of Eqs. (11a) and (11b). If $J=1$, the sparse matrix reduces to a tridiagonal matrix, which has been encountered in twodimensional models.

In the case of Fig. 3(c), approximate sidewall boundary conditions set $u_{m, 0}=u_{m, 1}$ and $u_{m, 4}=u_{m, 3}$. Following the same procedure as in the previous case, Eq. (A10) can also be transformed into a sparse matrix similar to Eq. (A11). The new sparse matrix has only five diagonals without the block matrices $G_{m, 1}$ and $F_{m, 3}$ occurring at the corners. The different block matrices are given in Eq. (12).

${ }^{1}$ F. B. Jensen, W. A. Kuperman, M. B. Porter, and H. Schmidt, Computational Ocean Acoustics (AIP, New York, 1994), Chap. 6.

${ }^{2}$ M. F. Levy and A. A. Zaporozhets, "Target scattering calculations with the parabolic equation method," J. Acoust. Soc. Am. 103, 735-741 (1998).

${ }^{3}$ G. J. Orris and M. D. Collins, "The spectral parabolic equation and threedimensional backscattering,"’ J. Acoust. Soc. Am. 96, 1725-1731 (1994). 
${ }^{4}$ D. Lee, G. Botseas, and W. L. Siegmann, "Examination of threedimensional effects using a propagation model with azimuth-coupling capability (FOR3D),', J. Acoust. Soc. Am. 91, 3192-3202 (1992).

${ }^{5}$ M. D. Collins and S. A. Chin-Bing, "A three-dimensional parabolic equation model that includes the effects of rough boundaries," J. Acoust. Soc. Am. 87, 1104-1109 (1990).

${ }^{6}$ A. T. Abawi, W. A. Kuperman, and M. D. Collins, "The coupled mode parabolic equation,' J. Acoust. Soc. Am. 102, 233-238 (1997).

${ }^{7}$ G. A. Athanassoulis and A. M. Prospathopoulos, "Three-dimensional acoustic scattering of a source-generated field from a cylindrical isliand,", J. Acoust. Soc. Am. 100, 206-218 (1996).

${ }^{8}$ D. Zhu and L. Bjørnø, "A hybrid 3-D, two-way IFD PE model for 3-D acoustic backscattering,' J. Comput. Acoust. 7(2), 133-145 (1999).

${ }^{9}$ M. D. Collins and R. B. Evans, "A two-way parabolic equation for acoustic backscattering in the ocean,', J. Acoust. Soc. Am. 91, 1357-1368 (1992).

${ }^{10}$ D. Lee and M. H. Schultz, Numerical Ocean Acoustic Propagation in Three Dimensions (World Scientific, Singapore, 1995), Chaps. 4 and 5.

${ }^{11}$ W. H. Press, S. A. Teukolsky, W. T. Vetterling, and B. P. Flannery, Numerical Recipes, 2nd ed. (Cambridge U. P., Cambridge, 1992), Chap. 2.

${ }^{12}$ R. B. Evans, "A coupled mode solution for acoustic propagation in a waveguide with stepwise depth variations of a penetrable bottom," J. Acoust. Soc. Am. 74, 188-195 (1983). 